\title{
THE CHOQUET REPRESENTATION IN THE COMPLEX CASE $^{1}$
}

\author{
BY R. R. PHELPS
}

1. Introduction and review. In its geometrical form, the Choquet representation theorem can be viewed as an infinite dimensional generalization of a classical theorem of Minkowski concerning finite dimensional compact convex sets. Indeed, suppose that $K$ is a compact convex subset of a locally convex Hausdorff real topological vector space $E$. If $E$ is assumed to be finite dimensional, then the Minkowski theorem asserts that each point $x$ in $K$ is a convex combination (or barycenter) of some finite set of extreme points; that is, there exist positive real numbers $a_{1}, a_{2}, \ldots, a_{n}$ and points $x_{1}, x_{2}, \ldots, x_{n}$ in ext $K$, the set of extreme points of $K$, such that $\Sigma a_{k}=1$ and $x=\Sigma a_{k} x_{k}$. Furthermore, each point of $K$ admits exactly one such representation if and only if $K$ is a simplex. If $E$ is assumed to be infinite dimensional, then the Minkowski theorem fails, although the Krein-Milman theorem does show that such convex combinations of extreme points are dense in $K$. If $K$ is metrizable, then the Choquet theorem applies (and says more than this): Each point in $K$ is the barycenter (precise definition below) of a Borel probability measure on the $G_{\delta}$ set ext $K$. Moreover, it is still true that each point of $K$ admits a unique such representation if and only if $K$ is a "simplex" (definition below).

The details of the relationships between the above results, together with some of their applications to real analysis, probability theory, functional analysis, etc., may be found in [19]. Additional general references for these and other results left unproved in what follows are [1], [3], [20], [21].

In order to formulate the representation theorem for nonmetrizable compact convex $K$, we need to introduce some definitions. If $X$ is a compact Hausdorff space, let $M(X)$ denote the space of all complex valued finite regular Borel measures on $X$. In what follows, the word "measure" will always mean an element of $M(X)$. Let $P(X)$ denote the convex set of all probability measures in $M(X)$, i.e., those positive measures of total mass 1 . If $K$ is a compact convex subset of the locally convex space $E$, then for each $\mu$ in $P(K)$ there exists a unique point $x$ in $K$ (the resultant, or barycenter of $\mu$ ) which is characterized by the relation

$$
f(x)=\int_{K} f d \mu \quad(\equiv \mu(f)) \text { for each } f \text { in } E^{*} .
$$

Equivalently, $h(x)=\mu(h)$ for each $h$ in $A(K)$, the space of all affine real-valued continuous functions on $K$. If $x$ is the resultant of some $\mu$ from $P(K)$, we

An invited address presented at the 710th meeting of the American Mathematical Society, Tucson, Arizona, November 24, 1973; received by the editors December 10, 1975.

AMS (MOS) subject classifications (1970). Primary 28A10, 28A45, 46E15.

Key words and phrases. Compact convex sets, Choquet's theorem, simplexes, affine functions, complex Borel measures.

'Work supported in part by a grant from the NSF. 
write $x=r(\mu)$ and we say that $\mu$ represents $x$, or is a representing measure for $x$. There is an important partial ordering on $P(K)$ defined as follows: We say $\mu>\lambda$ provided $\mu(f) \geqslant \lambda(f)$ for each continuous convex function $f$ on $K$. If $f$ is an affine continuous function on $K$, then both $f$ and $-f$ are convex; it follows that if $\mu>\lambda$, then $r(\mu)=r(\lambda)$. The weak* compactness of $P(K)$ yields the fact that any measure $\mu$ in $P(K)$ (in particular, any point mass) is dominated by a maximal measure from $P(K)$. (The terms "dominated" and "maximal" refer to the above ordering.) Thus, if $x \in K$, then there always exists a maximal probability measure $\mu$ dominating the point mass $\varepsilon_{x}$; since $r(\mu)=r\left(\varepsilon_{x}\right)=x$, the measure $\mu$ has $x$ as its barycenter. A detailed study of the support of a maximal measure then leads to the following result, the geometrical form of the representation theorem.

TheOREM 1.1 (ChOQUeT-Bishop-DE LeEuw). Suppose that $K$ is a compact convex subset of a locally convex space. If $x \in K$, then there exists a (maximal) probability measure $\mu$ on $K$ which has $x$ as its barycenter and which vanishes on every Baire subset of $K \backslash$ ext $K$.

As we mentioned above, if $K$ is metrizable, then ext $K$ is a $G_{\delta}$ set; in particular, it is a Baire set and, hence, any maximal measure is supported by ext $K$. (Even when $K$ is not metrizable, any probability measure with support contained in ext $K$ is maximal.) If $K$ is not metrizable, then ext $K$ need not be a measurable set and examples show that the above result is essentially the best that can be expected. The existence of such thorny examples forces us to walk a different path; we must give up hope of always finding representing measures which sit on ext $K$, and we focus instead on maximal measures. This is most clearly evident in the Choquet-Meyer uniqueness theorem; an elegant result, it is formulated purely in terms of maximal measures. Before stating it, we need to say what is meant by an infinite dimensional simplex.

Definition. A convex subset $K$ of a locally convex space $E$ is said to be a simplex provided the cone generated by $K \times\{1\}$ in the space $E \times R$ is a lattice in the partial ordering which it induces on $E \times R$.

This definition (rather abstract at first sight) can be reformulated in the following more geometrical way: A bounded closed convex set $K$ is a simplex if and only if the intersection of any two homothetic images of $K$ (that is, any two sets of the form $\alpha K+x, \alpha>0, x \in E$ ) is either empty, a single point, or a homothetic image of $K$. If $E$ is finite dimensional and $K$ is compact, then this is, in turn, equivalent to the assertion that $K$ is the convex hull of a finite and affinely independent set of points, that is, $K$ is a simplex in the usual sense.

Theorem 1.2 (ChOQuet-Meyer). Let $K$ be a compact convex subset of a locally convex space. Each point of $K$ is the barycenter of a unique maximal probability measure if and only if $K$ is a simplex.

The geometric version of the existence theorem fits most naturally into the context of real linear spaces; it is only when one puts it into the "analytic" form described below that the motivation for a complex version becomes apparent.

Let $X$ be a compact Hausdorff space and denote by $C_{R}(X)$ [resp. $C(X)$ ] the Banach space of all real valued [resp. complex valued] continuous 
functions on $X$, in the supremum norm. Suppose that $A$ is a linear subspace of $C_{R}(X)$ or of $C(X)$ and that $A$ separates the points of $X$. We will occasionally need to assume that $A$ is uniformly closed. If $A$ contains the constant functions (equivalently, if $1 \in A$ ), we define the state space $S_{A}$ of $A$ to be $\left\{L \in A^{*}:\|L\|=1\right.$ and $\left.L(1)=1\right\}$. This is a weak* compact convex subset of $A^{*}$ and (considering $A^{*}$ as a vector space over the real numbers) the existence and uniqueness theorems can be applied to $S_{A}$. Before actually doing this, note that the evaluation map $\phi: X \rightarrow A^{*}$ defined by $\phi(x)(f)=$ $f(x), x \in X, f \in A$, is a homeomorphism of $X$ into the weak* compact unit ball $U$ of $A^{*}$; in fact, into $S_{A}$ if $1 \in A$. Moreover, $\phi(X)$ contains ext $S_{A}$, so the following definition of the Choquet boundary $\partial A$ makes sense: Let $\partial A=$ $\phi^{-1}\left(\right.$ ext $\left.S_{A}\right)$ be the set of all points in $X$ which give rise to extreme evaluation functionals. This set is a "boundary" for $A$ in the sense that every function in $A$ attains its maximum absolute value in at least one point of $\partial A$ (and perhaps elsewhere). The smallest closed set with this property is the well-known Silov boundary; it is equal to the closure of $\partial A$. If $L \in S_{A}$, then the representation theorem yields a maximal probability measure on $S_{A}$ which has $L$ as its barycenter. Since we are working in the weak* topology, the dual space of $A^{*}$ is $A$. Moreover [19, p. 30], maximal measures on $S_{A}$ are supported by the compact set $\phi(X)$ (in fact, by the closure of ext $S_{A}$ ) so they can be carried via this homeomorphism into measures on $X$. The above facts can easily be combined to yield the following analytic version of the existence and uniqueness theorems for the metrizable case.

THEOREM 1.3. Suppose that $X$ is a compact metric space and that $A$ is a linear subspace of $C_{R}(X)$ which separates points and contains the constants. To each $L \in S_{A}$ there corresponds a probability measure $\mu$ on $X$ such that

$$
\mu(\partial A)=1 \text { and } L(f)=\int_{X} f d \mu \quad(f \in A) .
$$

Each $L \in S_{A}$ admits a unique such measure if and only if $S_{A}$ is a simplex.

This result remains valid for nonmetric $X$ if the condition that $\mu(\partial A)=1$ is replaced by the condition that $\mu$ be a boundary measure (an appropriate maximality property which will be defined in $\$ 2$ ). The above theorem is more than a corollary to the geometric version, since it actually implies the latter. Indeed, if $K$ is a compact convex subset of $E$, take $A$ to be the subspace $A(K)$ of $C_{R}(K)$ consisting of all continuous affine functions on $K$. It is readily verified that $\phi: K \rightarrow S_{A}$ is an affine homeomorphism of $K$ onto $S_{A}$ and that $\phi(\partial A)=\operatorname{ext} S_{A}$. This, together with the fact that (the restrictions to $K$ of) the functions of the form $f+r\left(f \in E^{*}, r\right.$ real $)$ are uniformly dense in $A(K)$ [19, Proposition 4.5] makes it easy to deduce the geometric version from the above analytic version.

The following characterization of the Choquet boundary is occasionally useful.

Proposition 1.4. A point $x \in X$ is in the Choquet boundary $\partial A$ for $A$ if and only if the only probability measure on $X$ which represents the linear functional $f \rightarrow f(x)(f \in A)$ is the point mass $\varepsilon_{x}$. Equivalently, a point $x$ of a compact convex set $K$ is an extreme point if and only if $\varepsilon_{x}$ is the only probability measure on $K$ having $x$ as its barycenter. 
The analytic version of the representation theorem was first introduced by Bishop and de Leeuw in 1959 in order to obtain boundary measures which represent continuous linear functionals on a uniform algebra $A \subseteq C(X)$, that is, on a uniformly closed subalgebra $A$ of $C(X)$ which contains the constant functions and separates points of $X$. Of course, it only gives such measures for functionals in $S_{A}$. In order to represent an arbitrary functional $L$ in $A^{*}$, they utilized the Riesz representation theorem and the Hahn decomposition to produce a complex representing measure which vanished on the Baire subsets of $X \backslash \partial A$, but which could possibly have its norm as great as $4\|L\|$. More than ten years later, Hustad [11] proved that it is possible to find such a complex representing measure of norm $\|L\|$ (the smallest possible norm) and Hirsberg [9] showed that Hustad's method actually yields a boundary measure. Hustad's theorem makes the question of uniqueness a meaningful one: For which $A \subseteq C(X)$ is it true that every $L \in A^{*}$ admits a unique complex representing measure $\mu$ which is a boundary measure and which satisfies $\|L\|=\|\mu\|$ ? This was answered in [8] for the case $1 \in A$, and an existence theorem was proved for the general case. Choquet [4], [5] independently used different methods to prove an existence theorem for the general case, and he formulated an appropriate version of the uniqueness theorem.

The remainder of this paper is devoted to the general existence and uniqueness theorems. The proof of the latter is given in somewhat more detail, since it has not previously appeared in print. We have benefitted considerably from our correspondence with $\mathrm{G}$. Choquet on this subject and from our conversations with R. Fuhr; we are grateful to both of them. We also thank S. Fitzpatrick for his helpful comments.

2. The existence theorem. In what follows, $X$ will be a compact Hausdorff space and $A$ will be a point-separating complex linear subspace (not necessarily uniformly closed) of $C(X)$. Since we do not assume that $1 \in A$, we cannot define the state space $S_{A}$ of $A$, as was done in $\S 1$. In its place we work with the weak* compact convex unit ball

$$
U=\left\{L \in A^{*}:\|L\| \leqslant 1\right\}
$$

of $A^{*}$. It is easy to see that the evaluation map $\phi: X \rightarrow A^{*}$ defined in $\S 1$ is still a homeomorphism, with values in $U$. We let $T$ denote the unit circle in the complex plane:

$$
T=\{t \in \mathbf{C}:|t| \leqslant 1\} .
$$

All the complications which arise in the complex versions of the existence and uniqueness theorems come from the fact that both $U$ and ext $U$ are invariant under multiplication by elements of $T$. A proof of the following elementary, but important, fact may be found in [6, p. 441].

LEMMA 2.1. If $L$ is an extreme point of $U$, then there exist $x \in X$ and $t \in T$ (not necessarily unique) such that $L=t \phi(x)$. Equivalently, ext $U \subseteq T \phi(X)$.

Obviously, if $L \in \operatorname{ext} U$, then $t L \in$ ext $U$ for all $t \in T$. It follows from this and the above lemma that for each extreme point $L$, the "circle" $T L$ of extreme points intersects $\phi(X)$, so $\phi^{-1}(\operatorname{ext} U)$ is nonempty and the following definition makes sense.

Definition. The Choquet boundary of $A$ is defined to be the subset 
$\phi^{-1}$ (ext $U$ ) of $X$ and is denoted by $\partial A$. A complex measure $\mu$ in $X$ is said to be a boundary measure if its total variation $|\mu|$ is either the zero measure, or when carried via $\phi$ to a positive measure $|\mu| \circ \phi^{-1}$ on $U$, is maximal in the sense defined in $\S 1$. The image $|\mu| \circ \phi^{-1}$ of $|\mu|$ under $\phi$ is also denoted by $\phi(|\mu|)$.

If $1 \in A$, the above definition of $\partial A$ coincides with the one given in $\S 1$. Note that Lemma 2.1 and the subsequent remarks imply that ext $U=$ $T \phi(\partial A)$. To see that the term "boundary measure" is a reasonable one, suppose that $X$ is a compact metric space. Then $U$ is metrizable in the weak* topology and both ext $U$ and $\partial A$ are $G_{\delta}$ sets. When combined with the remarks following Theorem 1.1, the formula

$$
\phi(|\mu|)(\operatorname{ext} U)=|\mu|\left(\phi^{-1}(\operatorname{ext} U)\right)=|\mu|(\partial A)
$$

shows that in the metric case $\mu$ is a boundary measure if and only if it is supported by the Choquet boundary $\partial A$.

THEOREM 2.2 (EXISTENCE). Let $X$ be a compact Hausdorff space and suppose that $A$ is a point-separating subspace of $C(X)$. To each $L_{0}$ in $A^{*}$ there corresponds a complex measure $\mu$ on $X$ such that

(a) $\|\mu\|=\left\|L_{0}\right\|$,

(b) $L_{0}(f)=\int_{X} f d \mu$ for each $f \in A$, and

(c) $\mu$ is a boundary measure.

The proof will be given somewhat informally. Note first that there is no loss of generality in assuming that $\left\|L_{0}\right\|=1$, and we will do that throughout. The basic ideas of the proof are best illustrated by carrying it out for the special case when $L_{0}$ is actually a convex combination of extreme points of $U$. In that case,

$$
L_{0}=\sum_{k=1}^{n} a_{k} L_{k}, \quad a_{k}>0, \quad \sum_{1}^{n} a_{k}=1, \quad L_{k} \in \operatorname{ext} U, \quad k=1,2, \ldots, n .
$$

By Lemma 2.1 we can express each of the extreme points $L_{k}$ in the form $L_{k}=t_{k} \phi\left(x_{k}\right)$ for some $t_{k} \in T, x_{k} \in \partial A$. Define $\mu=\sum_{k=1}^{n} a_{k} t_{k} \varepsilon_{x_{k}}$; this is clearly supported by $\partial A$, hence is a boundary measure. Since $|\mu|=\sum a_{k} \varepsilon_{x_{k}}$ is a probability measure, we have $\|\mu\|=1=\left\|L_{0}\right\|$. Finally, if $f \in A$, then

$$
\mu(f)=\sum a_{k} t_{k} f\left(x_{k}\right)=\sum a_{k} L_{k}(f)=L_{0}(f),
$$

so $\mu$ represents $L_{0}$.

The proof for an arbitrary functional $L_{0}$ of norm one is patterned after the above. We cannot expect to write $L_{0}$ as a convex combination of extreme points, but we can express it as the barycenter of a maximal probability measure $\lambda$ on $U$. This means that $\lambda(h)=h\left(L_{0}\right)$ for each continuous affine real valued function $h$ on $U$. If $f \in A$, then the function $L \rightarrow \operatorname{Re} L(f)$ is such an affine continuous map, as is $L \rightarrow \operatorname{Re} L(-i f)=\operatorname{Im} L(f)$. These two functions are the real and imaginary parts of the continuous affine complex valued function $\hat{f}$ defined by

$$
\hat{f}(L)=L(f), \quad L \in U .
$$

Since $\lambda$ is real we have 


$$
\lambda(\hat{f})=\lambda(\operatorname{Re} \hat{f})+i \lambda(\operatorname{Im} \hat{f})=\operatorname{Re} \hat{f}\left(L_{0}\right)+i \operatorname{Im} \hat{f}\left(L_{0}\right)=L_{0}(f) .
$$

If $X$ were metrizable, then $\lambda$ would sit on ext $U$. In general, any maximal measure sits on the closure of the extreme points $[19$, p. 30], so by virtue of Lemma 2.1, we know that $\lambda$ sits on the weak* compact set $T \phi(X)$. We would now like to mimic that part of the proof of the discrete case where we replaced each extreme point $L_{k}$ by $t_{k} \phi\left(x_{k}\right)$. This is done as follows. Let $\Phi$ be the continuous map from the compact product space $T \times X$ onto $T \phi(X)$ defined by

$$
\Phi(t, x)=t \phi(x), \quad t \in T, x \in X .
$$

If $\Phi$ were one-one (as it is, for instance, if $1 \in A$ ), then it would be a homeomorphism and we could identify $T \times X$ with $T \phi(X)$. Since, however, it is not generally one-one (we discuss this further in $\$ 3$ ), we use the easily proved fact [8, Lemma 7.2] that there exists a Borel measurable selection for $\Phi^{-1}$. Thus, for each $L \in T \phi(X)$ there exists $\left(t_{L}, x_{L}\right) \in T \times X$ such that $L=t_{L} \phi\left(x_{L}\right)$ and such that the map $L \rightarrow\left(t_{L}, x_{L}\right)$ is Borel measurable. (In particular, each of the component maps is Borel measurable.) Finally, we want to copy the last step of the proof of the discrete case, which consisted of representing each of the functionals $L_{k}$ in the form $t_{k} \phi\left(x_{k}\right)$ and then letting $\mu$ be the complex measure on $X$ which assigns "mass" $a_{k} t_{k}$ to the point $x_{k}$. We do this by letting $\mu$ be the complex measure $H \lambda$, where the latter is defined, for each $g \in C(X)$, by

$$
H \lambda(g)=\int_{T \phi(X)} t_{L} g\left(x_{L}\right) d \lambda(L) .
$$

This definition (due to Hustad [9]) makes sense, since for each such $g$, the map $L \rightarrow t_{L} g\left(x_{L}\right)$ is bounded and Borel measurable. Moreover, an easy computation shows that in the discrete case it gives exactly the same measure $\mu$ obtained earlier.

It remains to show that the measure $\mu=H \lambda$ satisfies conditions (a), (b) and (c) of Theorem 2.2. To see the first of these, namely, that $\|\lambda\|=1$, note that if $g \in C(X)$ and $|g| \leqslant 1$, then

$$
|\mu(g)| \leqslant \int_{T \phi(X)}\left|g\left(x_{L}\right)\right| d \lambda(L) \leqslant 1
$$

so, as a linear functional on $C(X)$, the norm of $\mu$ is at most 1 . On the other hand, once we have shown part (b), we will know that the restriction of $\mu$ to the subspace $A$ is the norm 1 functional $L_{0}$, so $\mu$ has norm exactly equal to 1 . To see part (b), recall that we showed earlier that $L_{0}(f)=\lambda(\hat{f})$ whenever $f \in A$. Since $\hat{f}$ is actually the restriction to $U$ of a linear functional, we also have $\hat{f}(t \phi(x))=t \hat{f}(\phi(x))=t f(x)$ for any $t \in T, x \in X$. It follows that for $f \in A$,

$$
\begin{aligned}
\mu(f) & =H \lambda(f)=\int_{T_{\phi}(X)} t_{L} f\left(x_{L}\right) d \lambda(L) \\
& =\int_{T \phi(X)} \hat{f}\left(t_{L} \phi\left(x_{L}\right)\right) d \lambda(L)=\lambda(\hat{f})=L_{0}(f) .
\end{aligned}
$$

To prove part (c), we must show that the probability measure $\phi(|\mu|)$ is maximal on $U$. This is a mildly technical result which is proved in detail in [8, Theorem 7.3], using a lemma from [7]. In the special case when $X$ is 
metrizable, it is easily shown as follows. Recall that in this case, $U$ is itself metrizable (in the weak* topology), so by Choquet's original representation theorem, the maximal measure $\lambda$ sits on the $G_{8}$ set ext $U=T \phi(\partial A)$. Consequently, the formula for $\mu$ becomes

$$
\mu(g)=\int_{T \phi(\partial A)} t_{L} g\left(x_{L}\right) d \lambda(L), \quad g \in C(X),
$$

and the corresponding total variation measure $|\mu|$ is given by

$$
|\mu|(g)=\int_{T_{\phi}(\partial A)} g\left(x_{L}\right) d \lambda(L), \quad g \in C(X) .
$$

This formula is actually valid for any bounded Borel measurable function $g$ [8, Lemma 3.7]. Thus, if $g$ denotes the characteristic function of $\partial A$ we obtain

$$
|\mu|(\partial A)=|\mu|(g)=\int_{T \phi(\partial A)} g\left(x_{L}\right) d \lambda(L)=1,
$$

where the last equality depends on the fact that if $L \in T \phi(\partial A)$, then the point $x_{L}$ is in $\partial A$ and hence $g\left(x_{L}\right)=1$. It is immediate from $|\mu|(\partial A)=1$ that $\phi(|\mu|)$ is supported by $\phi(\partial A)$ and is therefore maximal.

There is a refinement of the existence theorem which seems to be potentially useful in applications. In the real case it was first proved by Rao [22] (see, also, [16], [20], [24]) and may be formulated as follows: Assume that $X$ is a compact metric space and that the point-separating subspace $A$ of $C_{R}(X)$ contains the constants. For each $x \in X$ it is possible to choose a probability measure $\mu_{x}$ (which represents evaluation at $x$ and sits on $\partial A$ ) in such a way that the map $x \rightarrow \mu_{x}$ from $X$ into the weak* compact metrizable space $P(X)$ is Borel measurable. (This is equivalent to saying that for each $f \in C_{R}(X)$, the real valued function $x \rightarrow \mu_{x}(f)$ is Borel measurable.) P. Saab [23] has extended Rao's theorem to the complex case, without assuming that $1 \in A$. Recall that $M(X)$ denotes the space of complex finite regular Borel measures on $X$.

THEOREM 2.3 (RAO-SAAB). Suppose that $X$ is a compact metric space and that $A$ is a point-separating subspace of $C(X)$. There exists a Borel measurable map $x \rightarrow \mu_{x}$ from $X$ into the weak* compact metrizable unit ball of $M(X)$ such that, for each $x \in X$,

(i) $\mu_{x}(f)=f(x)$ for all $f$ in $A$, and

(ii) $\left|\mu_{x}\right|(\partial A)=1=\left\|\mu_{x}\right\|$.

The idea of the proof is easy; one simply composes several Borel measurable maps. First, $x \rightarrow \phi(x)$ is continuous from $X$ into $U$. Next, Rao's theorem is applicable to the (weak*) compact convex set $U$ (and the subspace of affine functions in $C_{R}(U)$ ), so there exists a measurable map $L \rightarrow \lambda_{L}$, where $\lambda_{L}$ is a probability measure on ext $U$ with barycenter $L$. Finally, Hustad's map is continuous and carries $\lambda_{L}$ into a complex boundary measure $H\left(\lambda_{L}\right)$ representing $L$, so the composition $x \rightarrow \mu_{x}=H\left(\lambda_{\phi(x)}\right)$ yields the desired map.

3. Uniqueness. Suppose, as before, that $A$ is a point-separating linear subspace of $C(X)$. We will say that uniqueness holds for $A$ provided that for each $L \in A^{*}$ there exists a unique boundary measure $\mu$ on $X$ which represents $L$ and for which $\|\mu\|=\|L\|$. Recall that in the case when the scalars are real, uniqueness is nicely characterized in terms of the state space $S_{A}$ : Uniqueness 
holds (in the real case) if and only if $S_{A}$ is a simplex. This result suggests an analogous characterization for the complex case, since we can still define $S_{A}$ (at least when $1 \in A$ ). It turns out that while $S_{A}$ is necessarily a simplex if uniqueness holds, the converse is false [8]. In order to obtain a geometrical criterion for uniqueness we must use the entire unit ball $U$.

Definition. A convex subset $F$ of a convex set $K$ is said to be a face of $K$ provided $F$ is nonempty and the following holds: If $x, y \in K$ and $\alpha x+$ $(1-\alpha) y \in F$ for some $0<\alpha<1$, then $x, y \in F$. A convex set $K$ is said to be a simplexoid if every proper face of $K$ is a simplex.

The simplest example of a face (other than $K$ itself) is the set $F$ of all points where a nonconstant affine real-valued function on $K$ attains its maximum (provided, of course, that $F$ is nonempty). A convex polytope in Euclidean 3-space is a simplexoid if and only if all of its two-dimensional faces are plane triangles. Other examples of simplexoids are the unit ball in Euclidean $n$-space and the (weakly compact) unit ball in Hilbert space.

The following theorem was originally proved in [8]; it will be obtained here as a corollary to Theorem 3.3 (below).

THEOREM 3.1. Suppose that $X$ is compact Hausdorff and that the point-separating subspace $A$ of $C(X)$ contains the constant functions. Then uniqueness holds for $A$ if and only if the unit ball $U$ of $A^{*}$ is a simplexoid.

Since the above result does not involve the state space of $A$, it would seem reasonable to expect that the same criterion for uniqueness would remain valid even if $1 \notin A$. Very simple examples, however, show that this is not the case. The problem is that arbitrary subspaces of $C(X)$ are too general. Suppose, for example, that $E$ is a complex Banach space and let $X$ be the unit ball of $E^{*}$, in its weak* topology. We can easily identify $E$ with the uniformly closed subspace $A$ of $C(X)$ consisting of all complex continuous affine functions $f$ on $X$ which satisfy $f(t x)=t f(x)$ for each $x \in X, t \in T$. In this case, the map $\phi: X \rightarrow U$ is an affine homeomorphism and no matter what geometrical conditions are satisfied by the dual ball $U$ of $A$, uniqueness fails for every $L \in \operatorname{ext} U$. Indeed, any such $L$ is of the form $\phi(x)$, where $x$ is an extreme point of $X$, and for any $t \in T$ the complex measure $\mu_{t}=\bar{t}_{t x}$ has norm one and is supported by the extreme point $t x$, hence is a boundary measure. Moreover, if $f \in A$, then $\mu_{t}(f)=\bar{t} f(t x)=f(x)=L(f)$, so each $\mu_{t}$ represents $L$.

Choquet [4] has suggested a way to bypass such examples, by relaxing the uniqueness requirement. The idea is to demand only that any two boundary measures which represent $L$ and have norm $\|L\|$ be equivalent, in the sense defined below.

Definition. Let $X$ be a compact Hausdorff space and suppose that $A$ is a point-separating linear subspace of $C(X)$. Let $A^{\sim}$ denote the set of all $g$ in $C(X)$ such that $s g(x)=\operatorname{tg}(y)$ whenever $s, t \in T, x, y \in X$ and $s f(x)=$ $t f(y)$ for all $f \in A$. If $\lambda, \mu$ are two complex regular Borel measures on $X$, we write $\lambda \approx \mu$ provided $\lambda(g)=\mu(g)$ for each $g \in A^{\sim}$. (This will generally be applied to measures of norm one.)

Clearly, $A^{\sim}$ is a uniformly closed linear subspace of $C(X)$ which contains $A$, and the relation $\approx$ is an equivalence relation depending on $A$. Recall that the map $\Phi: T \times X \rightarrow T \phi(X) \subseteq A^{*}$ was defined by $\Phi(t, x)=t \phi(x)$. It is 
immediate that $g \in A^{\sim}$ if and only if $\operatorname{sg}(x)=\operatorname{tg}(y)$ whenever $\Phi(s, x)=$ $\Phi(t, y)$. In the example of nonuniqueness described above, where the infinitely many distinct boundary measures $\mu_{t}$ represented evaluation at $x$, we have $\mu_{t} \approx \varepsilon_{x}$ for each $t$. Indeed, the identity $\bar{t} \phi(t x)=\phi(x)$ shows that for each $g \in A^{\sim}$ we must have $\bar{t} g(t x)=g(x)$, which is the same as $\mu_{t}(g)=\varepsilon_{x}(g)$, that is, $\mu_{t} \approx \varepsilon_{x}$ for each $t$. Thus, the measures $\mu_{t}$ are all the same, modulo $\approx$.

Definition. If $A$ is a point-separating subspace of $C(X)$, we say that uniqueness holds (modulo $\approx$ ) for $A$ provided the following is true: If $L \in A^{*}$, $\|L\|=1$, and if $\lambda, \mu$ are complex boundary measures of norm 1 on $X$ which represent $L$, then $\lambda \approx \mu$.

THEOREM 3.2. In order that uniqueness hold (modulo $\approx$ ) for $A$ it is necessary and sufficient that each $L$ of norm 1 in $A^{*}$ be the barycenter of a unique maximal probability measure on $U$.

This result will subsequently be reformulated as follows.

Theorem 3.3. Uniqueness holds (modulo $\approx$ ) for $A$ if and only if $U$ is $a$ simplexoid.

These theorems, formulated by Choquet, have maintained the geometric flavor and simplicity of Theorem 3.1 by sacrificing uniqueness. Since we are primarily interested in the latter property, it is important that we give conditions under which equivalence of two measures implies that they are, in fact, the same. We do this next, postponing the proof of Theorem 3.2 temporarily. The following proposition has also been observed by Choquet [5].

Proposition 3.4. The following six assertions are equivalent.

(i) If $\lambda, \mu$ are measures of norm one on $X$ satisfying $\lambda \approx \mu$, then $\lambda=\mu$.

(ii) The map $\Phi$ is one-one.

(iii) $A^{\sim}=C(X)$.

(iv) $1 \in A^{\sim}$.

(v) There exists a strictly positive function in $A^{\sim}$.

(vi) $\{|f|: f \in A\}$ separates points of $X$.

If $A$ is a subalgebra of $C(X)$, then $A$ satisfies each of the above.

Proof. The implications (i) $\rightarrow$ (ii) $\rightarrow$ (iii) $\rightarrow$ (iv) $\rightarrow$ (v) are either obvious or follow readily from the definitions, as do (vi) $\rightarrow$ (ii) and (iii) $\rightarrow$ (i).

(v) implies (ii). Suppose that $s \phi(x)=t \phi(y)$ and that $f \in A^{\sim}$ is strictly positive. Then $f(x)=\bar{s} t f(y)$ and therefore $\bar{s} t>0$. Since $\bar{s} t \in T$, this shows that $\bar{s} t=1$ so $s=t$ and hence $\phi(x)=\phi(y)$, which implies that $x=y$.

(ii) implies (vi). Suppose that $x, y \in X$ and that $|f(x)|=|f(y)|$ for all $f \in A$. We want to show that $x=y$. In view of (ii), it suffices to show that there exists $t \in T$ such that $f(x)=t f(y)$ for all $f \in A$. If $f(y)=0$, then $f(x)=0$, so we need only verify that for $f, g \in A$ with $f(y) \neq 0 \neq g(y)$, we have $f(x) / f(y)=g(x) / g(y)$; since these ratios have modulus 1 , their common value will be the desired number $t$. The function $h=f(y) g-g(y) f$ is in $A$ and vanishes at $y$; since $|h(x)|=|h(y)|=0$, we have $h(x)=0$, exactly what was needed.

Finally, if $A$ is a subalgebra of $C(X)$ and $s \phi(x)=t \phi(y)$, then for all $f \in A$ we have $s f(x)=t f(y)$ and $s f^{2}(x)=t f^{2}(y)$ (since $\left.f^{2} \in A\right)$. If $f(x)=0$ for all 
$f \in A$, then $f(y)=0$ as well, hence $x=y$ and therefore $s=t$. If $f_{1}(x) \neq 0$ for at least one $f_{1} \in A$, then the above two equations yield $f_{1}(x)=f_{1}(y)$, so $s=t$ and hence $f(x)=f(y)$ for all $f \in A$, again giving $x=y$.

The proof of Theorem 3.2 will be greatly simplified by reformulating the notion of equivalence of two measures; this requires some preliminary definitions.

Definition. Suppose that $g \in C(U)$, where $U$ is the weak* compact unit ball of $A^{*}$. Define the "homogeneous part" of $g$ to be the function hom $g$, where for $L \in U$ we set

$$
(\operatorname{hom} g)(L)=\int_{T} \bar{t} g(t L) d t \text {. }
$$

(Integration is with respect to Haar measure on the circle $T$.) It is not hard to see that hom $g \in C(U)$, that $\|$ hom $g\|\leqslant\| g \|$ and that (hom $g)(t L)=$ $t$ (hom $g)(L)$ for each $t \in T, L \in U$, which means that hom $g$ is $T$-homogeneous. Obviously, hom $g=g$ if and only if $g$ is $T$-homogeneous. Next, if $\mu$ is a complex regular Borel measure on $U$, define hom $\mu$ by

$$
(\operatorname{hom} \mu)(g)=\mu(\text { hom } g), \quad g \in C(U) \text {, }
$$

so hom $\mu$ is again a complex regular Borel measure on $U$, of norm at most $\|\mu\|$. It is useful in what follows to observe that if $\mu_{1}, \mu_{2}$ are two measures on $U$, then hom $\mu_{1}=$ hom $\mu_{2}$ if and only if $\mu_{1}(g)=\mu_{2}(g)$ for each $T$-homogeneous continuous function $g$ on $U$.

Proposition 3.5. Suppose that $\mu, \nu$ are complex measures on $X$. Then $\mu \approx \nu$ if and only if hom $\phi(\mu)=$ hom $\phi(\nu)$.

Proof. Suppose that hom $\phi(\mu)=$ hom $\phi(\nu)$; then $\phi(\mu)(g)=\phi(\nu)(g)$ for every continuous $T$-homogeneous function on $U$. We want to show that $\mu(f)=\nu(f)$ for each $f \in A^{\sim}$. Given such a function $f$, define $g$ on $C(T \phi(X))$ as follows: If $L \in T \phi(X)$, say $L=t \phi(x)$ for some $t \in T, x \in X$, then let $g(L)=t f(x)$. Since $f \in A^{\sim}$, this is independent of the representation of $L$. A routine argument, using the compactness of $T$ and $X$, shows that $g$ is continuous. By the Tietze extension theorem there exists a continuous extension $g_{1}$ of $g$ to all of $U$, so that $g_{2}=$ hom $g_{1}$ is a continuous $T$-homogeneous function on $U$. Using the definition of hom $g_{1}$, it is readily seen that $g_{2}$ is an extension of $g$. By hypothesis, $\mu\left(g_{2} \circ \phi\right)=\phi(\mu)\left(g_{2}\right)=\phi(\nu)\left(g_{2}\right)=\nu\left(g_{2} \circ \phi\right)$. Moreover, if $x \in X$, then $\left(g_{2} \circ \phi\right)(x)=g_{2}(\phi x)=g(\phi x)=f(x)$, so $\mu(f)=$ $\nu(f)$, which completes the first part of the proof.

To prove the converse, suppose that $\mu \approx \nu$, so that $\mu(f)=\nu(f)$ for each $f \in A^{\sim}$. If $g$ is any $T$-homogeneous function in $C(U)$, it follows from the definitions that $g \circ \phi \in A^{\sim}$ and hence $\phi(\mu)(g)=\mu(g \circ \phi)=\nu(g \circ \phi)=$ $\phi(\nu)(g)$. Thus, $\phi(\mu)=\phi(\nu)$ on the $T$-homogeneous functions in $C(U)$, which is equivalent to hom $\phi(\mu)=$ hom $\phi(\nu)$.

We now prove the necessity portion of Theorem 3.2. Suppose, then, that $L \in A^{*},\|L\|=1$, and that $\lambda_{1}, \lambda_{2}$ are maximal probability measures on $U$ with barycenter $L$. Let $H$ be the map defined in the proof of Theorem 2.2 and let $\mu_{k}=H \lambda_{k}, k=1,2$. As indicated in that proof, each of the measures $\mu_{1}, \mu_{2}$ is a boundary measure of norm 1 which represents $L$, so by hypothesis we must have $\mu_{1} \approx \mu_{2}$. Proposition 3.5 shows that hom $\phi\left(\mu_{1}\right)=\operatorname{hom} \phi\left(\mu_{2}\right)$, and a straightforward computation, using the definition of $H$, shows that for any 
continuous $T$-homogeneous function $g$ on $U$ we must have $\phi\left(\mu_{k}\right)(g)=\lambda_{k}(g)$, $k=1,2$. This last equality implies that

$$
\text { hom } \lambda_{1}=\text { hom } \phi\left(\mu_{1}\right)=\text { hom } \phi\left(\mu_{2}\right)=\text { hom } \lambda_{2} \text {. }
$$

The proof of this half of the theorem will be complete once we have proved Lemma 3.6 below. We first require a definition.

Definition. Let $\nu$ be a complex measure on $U$ and express $\nu$ in its polar decomposition: $\nu=h|\nu|$, where $|\nu|$ is the total variation of $\nu$ and $h$ is a Borel function of modulus 1 . For any $g \in C(U)$ define

$$
(R \nu)(g)=\int_{U} g(h(L) \cdot L) d|v|(L)
$$

It is clear that $R \nu$ is a positive measure on $U$, with $\|R \nu\|=\|\nu\|=|\nu|(U)$. If $g \in C(U)$ is $T$-homogeneous, then $(R \nu)(g)=\nu(g)$, so that hom $R \nu=$ hom $\nu$. In particular, if $\|\nu\|=1$ and if $g=\hat{f}$, where $f \in A$, this equality shows that the resultant $L$ in $U$ of the probability measure $R \nu$ is the "barycenter" of the complex measure $\nu$, that is, $\nu(f)=f(L)$ for every affine continuous function $f$ on $U$.

LEMMA 3.6. Suppose that $\lambda$ is a probability measure on $U$ with barycenter $L$ of norm one. Then $R($ hom $\lambda)=\lambda$.

Proof. Let $\nu=$ hom $\lambda$. From the definition of hom $\lambda$ we have $\nu(g)=\lambda(g)$ for any $T$-homogeneous $g \in C(U)$; in particular, this holds for $g=\hat{f}$, where $f \in A$. Using the facts that $1=\|L\|=\sup \{|L(f)|: f \in A,\|f\|=1\}$ and that $\lambda(\hat{f})=L(f)$ for $f \in A$, we see that $\|\nu\|=1$. Thus, if we write $\nu=h|\nu|$, where $h$ is a Borel function on $U$ of modulus 1 , then (writing $\bar{h}$ for the complex conjugate of $h$ ) we have

$$
\begin{aligned}
1 & =\|\nu\|=|\nu|(1)=\nu(\bar{h})=(\operatorname{hom} \lambda)(\bar{h})=\lambda(\text { hom } \bar{h}) \\
& =\int_{U} \operatorname{hom} \bar{h} d \lambda=\int_{U} \int_{T} \bar{t} \bar{h}(t L) d t d \lambda(L) .
\end{aligned}
$$

Since $|\overline{t h}(t L)|=1$, Fubini's theorem shows that, almost everywhere with respect to the probability measure $d t \times d \lambda$,

$$
\operatorname{th}(t L)=1 \text { on } T \times U \text {. }
$$

Using successively the definition of $R$, the identity $|\nu|=\bar{h}$, the definition of hom $\lambda$, Fubini's theorem and the equality (*), we obtain, for any $g \in C(U)$,

$$
\begin{aligned}
(R \nu)(g) & =\int_{U} g(h(L) L) d|v|(L)=\int_{U} \bar{h}(L) g(h(L) L) d \nu(L) \\
& =\int_{T} \bar{t}\left[\int_{U} \bar{h}(t L) g(h(t L) t L) d \lambda(L)\right] d t \\
& =\int_{T} \int_{U} g(L) d \lambda(L) d t=\lambda(g),
\end{aligned}
$$

which shows that $R \nu=\lambda$ and proves the lemma.

We can now finish the necessity portion of Theorem 3.2, by showing that $\lambda_{1}=\lambda_{2}$. We know that hom $\lambda_{1}=$ hom $\lambda_{2}$, so by Lemma 3.6, we have $\lambda_{1}=$ $R\left(\right.$ hom $\left.\lambda_{1}\right)=R\left(\right.$ hom $\left.\lambda_{2}\right)=\lambda_{2}$.

We now turn to the sufficiency portion of the proof of Theorem 3.2. Suppose, then, that $\mu_{1}, \mu_{2}$ are boundary measures of norm 1 on $X$, each of 
which represents the same functional $L \in A^{*}$, where $\|L\|=1$. Let $\nu_{k}=\phi\left(\mu_{k}\right)$ and let $\lambda_{k}=R\left(\nu_{k}\right), k=1,2$. Each of the measures $\lambda_{1}, \lambda_{2}$ is a probability measure on $U$ and we have, for $k=1,2$,

$$
\lambda_{k}(g)=\nu_{k}(g) \text { for all continuous } T \text {-homogeneous } g \text { on } U \text {. }
$$

If we apply (1) to $g=\hat{f}, f \in A$, we get

$$
\lambda_{k}(\hat{f})=\nu_{k}(\hat{f})=\mu_{k}(\hat{f} \circ \phi)=\mu_{k}(f)=L(f),
$$

so both $\lambda_{1}, \lambda_{2}$ have the same barycenter $L \in U$ of norm 1 and therefore, by hypothesis, $\lambda_{1}=\lambda_{2}$. On the other hand, (1) shows that hom $\lambda_{k}=$ hom $\nu_{k}$, so we conclude that

$$
\text { hom } \phi\left(\mu_{1}\right)=\operatorname{hom} \nu_{1}=\operatorname{hom} \lambda_{1}=\operatorname{hom} \lambda_{2}=\operatorname{hom} \nu_{2}=\operatorname{hom} \phi\left(\mu_{2}\right) \text {. }
$$

By Proposition 3.5, this implies that $\mu_{1} \approx \mu_{2}$ and the proof is complete.

Theorem 3.3 is a consequence of Theorem 3.2, combined with the fact that $U$ is a simplexoid if and only if each $L \in U$ of norm 1 is the barycenter of a unique maximal measure [8, p. 12]. Theorem 3.1 follows from Theorem 3.3, together with Proposition 3.4 (iv).

The tools we have developed for the uniqueness theorems are readily applicable to proving, in the general complex case, the following characterization of the Choquet boundary. It uses (and is analogous to) the characterization for the real case given in Propositon 1.4.

Proposition 3.7. Suppose that $A$ is a point-separating subspace of $C(X), A$ point $x \in X$ is in the Choquet boundary $\partial A$ if and only if $\mu \approx \varepsilon_{x}$ whenever $\mu$ is a complex measure of norm one on $X$ satisfying $\mu(f)=f(x)$ for each $f \in A$.

Note that Proposition 3.4 gives a number of conditions under which we can replace $\mu \approx \varepsilon_{x}$ by $\mu=\varepsilon_{x}$; in particular, this is true if $1 \in A$.

Proof. Suppose first that $x \in \partial A$, that $\|\mu\|=1$ and that $\mu$ represents evaluation at $x$. We want to show that $\mu \approx \varepsilon_{x}$; by Proposition 3.5 it suffices to prove that hom $\phi(\mu)=$ hom $\phi\left(\varepsilon_{x}\right)=$ hom $\varepsilon_{\phi(x)}$. Now, the measure $\lambda=R \phi(\mu)$ is a probability measure on $U$ with barycenter $\phi(x) \in$ ext $U$, so by Proposition 1.4, we have $\lambda=\varepsilon_{\phi(x)}$. On the other hand, for any $\nu$ it is true that hom $R \nu=$ hom $\nu$, so we conclude from Lemma 3.6 and the above that hom $\phi(\mu)=$ hom $R \phi(\mu)=$ hom $\lambda=$ hom $\varepsilon_{\phi(x)}$, which completes the proof. For the converse, suppose that $x \in X \backslash \partial A$, so that $\phi(x) \notin$ ext $U$. By the Choquet-Bishop-de Leeuw theorem we can find a maximal probability measure $\lambda$ on $U$ with barycenter $\phi(x)$. If $\lambda$ were the same as the point mass at $\phi(x)$, we could [by writing $\phi(x)=\left(L_{1}+L_{2}\right) / 2, L_{1}, L_{2}$ distinct points of $U$, and then letting $\lambda^{\prime}=\left(\varepsilon_{L_{1}}+\varepsilon_{L_{2}}\right) / 2$ ] find a probability measure $\lambda^{\prime} \neq \lambda$ with $\lambda^{\prime} \succ \lambda$, contradicting the maximality of $\lambda$. Thus, $\lambda \neq \varepsilon_{\phi(x)}$. Since, by Lemma 3.6, $\lambda=R\left(\right.$ hom $\lambda$ ) and $\varepsilon_{\phi(x)}=R\left(\right.$ hom $\left.\varepsilon_{\phi(x)}\right)$, we must have hom $\lambda \neq$ hom $\varepsilon_{\phi(x)}$, which means there exists a $T$-homogeneous continuous function $g$ on $U$ such that $\lambda(g) \neq g(\phi(x))$. To complete the proof we want to find a measure $\mu$ of norm 1 on $X$ which represents evaluation at $x$ but which is not equivalent to $\varepsilon_{x}$. The proof of Theorem 2.2 shows that the measure $\mu=H \lambda$ will certainly have the first two properties, so we need only show that $\mu(f) \neq f(x)$ for some $f \in A^{\tilde{}}$. Define $f$ on $X$ by $f(y)=g(\phi(y))$, where $g$ is the function obtained above. The homogeneity of $g$ readily implies that $f \in A^{\sim}$ 
and that $\lambda(g)=(H \lambda)(f)=\mu(f)$, while $f(x)=g(\phi(x)) \neq \lambda(g)$, which was to be shown.

4. Related results. The notion of a simplexoid is closely related to what Effros [7] calls a $T$-simplex: The unit ball $U$ of $A^{*}$ (where $A$ is separating subspace of $C(X))$ is called a $T$-simplex provided hom $\mu=$ hom $\lambda$ whenever $\mu$ and $\lambda$ are probability measures on $U$ having the same barycenter $L \in U$. It follows from Theorems 3.2 and 3.3 and Proposition 3.5 that $U$ is a simplexoid if and only if hom $\mu=\operatorname{hom} \lambda$ whenever $\mu$ and $\lambda$ are probability measures on $U$ having the same barycenter $L \in U$ with $\|L\|=1$. Thus, we would expect the $T$-simplexes to form a proper subclass of the simplexoids, and this is indeed the case: if $1 \in A$, then $U$ is a $T$-simplex if and only if it is a simplexoid and $A$ is selfadjoint [8, Theorem 4.4], [10]. By considering a given complex Banach space $E$ as a subspace $A$ of $C(U), U$ the weak* compact unit ball of $E^{*}$, one can formulate Effros' result [7]: The set $U$ is a T-simplex if and only if $E$ is $a$ Lindenstrauss space, that is, the space $E^{*}$ is linearly isometric to some complex $L_{1}$ space. Such spaces $E$ have been thoroughly studied; see, for instance, [10], [12]-[15], [17], [18].

An interesting method of proof of Hustad's existence theorem in the metric case, using the notion of dilations, has been sketched by Briem [2, Theorem 1].

\section{REFERENCES}

1. E. M. Alfsen, Compact convex sets and boundary integrals, Ergebnisse Math. Grenzgebiete, Band 57, Springer-Verlag, New York, 1971.

2. E. Briem, Interpolation in subspaces $C(X)$, J. Functional Analysis 12 (1973), 1-12. MR 49 \#7745. 1630.

3. G. Choquet, Lectures on analysis, Vol. II, Benjamin, New York, 1969. MR 40 \#3253; 44, p.

4. , Frontière-module et représentation intégrale [Résumé], Séminaire Choquet, Univ. de Paris (1971/1973), No. 8, 4 pp.

5. Frontières et représentations intégrales relatives $a ̀$ un espace de fonctions continues á valeurs complexes, 1973 (handwritten manuscript).

6. N. Dunford and J. T. Schwartz, Linear operators. Part I: General theory, Interscience, New York and London, 1958. MR 22 \#8302.

7. E. G. Effros, On a class of complex Banach spaces, Illinois J. Math. 18 (1974), 48-59. MR 48 \#6890.

8. R. Fuhr and R. R. Phelps, Uniqueness of complex representing measures on the Choquet boundary, J. Functional Analysis 14 (1973), 1-27. MR 50 \# 14186.

9. B. Hirsberg, Représentations intégrales des formes linéaires complexes, C. R. Acad. Sci. Paris Sér A-B 274 (1972), A1222-A1224. MR 45 \#5727.

10. B. Hirsberg and A. J. Lazar, Complex Lindenstrauss spaces with extreme points, Trans. Amer. Math. Soc. 186 (1973), 141-150 (1974). MR 48 \#11996.

11. O. Hustad, A norm preserving complex Choquet theorem, Math. Scand. 29 (1971), 272-278. MR 48 \#852.

12. Intersection properties of balls in complex Banach spaces whose duals are $L_{1}$ spaces, Acta Math. 132 (1974), 283-313.

13. A. Lima, Intersection properties of balls and subspaces in Banach spaces, Univ. of Oslo, Inst. of Math. Preprint Series No. 3, 1974, 75 pp.

14. - An application of a theorem of Hirsberg and Lazar, Math. Scand. (to appear).

15. - Complex Banach spaces whose duals are $L_{1}$-spaces, Israel J. Math. 24 (1976), 59-72.

16. L. H. Loomis, Dilations and extremal measures, Advances in Math. 17 (1973), 1-13. MR 51 \#11062. 
17. N. Nielsen and G. Olsen, Complex preduals of $L_{1}$ and subspaces of $l_{\infty}^{n}(C)$, Univ. of Oslo, Inst. of Math. Preprint Series No. 3, 1976, 25 pp.

18. G. H. Olsen, On the classification of complex Lindenstrauss spaces, Math. Scand. 35 (1974), 237-258. MR 51 \#3868.

19. R. R. Phelps, Lectures on Choquet's theorem, Van Nostrand, Princeton, N. J., 1966. MR 33 \#1690.

20. - Lectures on Choquet's theorem, rev. ed., Ergebnisse Math. Grenzgebiete, Springer-Verlag, New York and Berlin (to appear).

21. - Integral representations for elements of convex sets, Studies in Math., Vol. 15, Studies in Functional Analysis, R. Bartle (editor), Math. Assoc. Amer. (1977).

22. Murali Rao, Measurable selections of representing measures, Quart. J. Math. Oxford Ser. (2) 22 (1971), 571-573. MR 45 \#9117.

23. P. Saab, Représentation intégrale dans des sous-espaces de fonctions à valeurs vectorielles, Séminaire Choquet, $14^{e}$ année, 1974-75, No. 23, $10 \mathrm{pp}$.

24. G. F. Vincent-Smith, Measurable selections of simplicial maximal measures, J. London Math. Soc. (2) 7 (1973), 427-428. MR 49 \# 1068.

Department of Mathematics, University of Washington, Seatrle, Washington 98195 